\title{
Documentação e Informação em Química
}

Reaç̧̃es de Nitroarenos e seus Derivados com Reagentes Organomagnesianos

Conteúdos

\begin{tabular}{l} 
1. Introdução \\
1.1 Reagentes Organomagnesianos \\
1.2 Nitroarenos \\
2. Reações de nitroarenos com reagentes \\
organomagnesianos \\
2.1 Arílicos \\
2.2 Alquilicos \\
2.4 Vinilicos \\
3. Preparação de reagentes organomagesianos \\
nitro-arilicos \\
4. Redução de nitroarenos funcionalizados \\
5. Conclusões e Perspectivas \\
\hline
\end{tabular}

1. Introdução

1.1 Reagentes Organomagnesianos
Os reagentes organomagnesianos ocupan uma posição central na sintese orgânica, contemplando a necessidade constante de criação selectiva de ligações carbono-carbono e carbono-

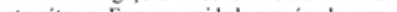
hor de continua de para as industrias farmacetica e agropuimica. Nos processos de sintese que envolvem reagentes de Grignard são usadas condiçôes relativamente suaves e altamente tolerantes à presença de uma grande variedade de grupos funcionais.

Em 1849, Frankland sintetizou a primeira molécula organometálica, o dimetilzinco. No entanto, é a Victor Grignard que se devem os maiores avanços nos primórdios da Química Organometálica. ${ }^{[2]}$ Hoje em dia, a química deste tipo de compostos é já um lugar comum, estando a sua preparação e sintese intensivamente
descrita.

\subsection{Nitroarenos}

Os substituintes nitro são normalmente introduzidos numa molécula aromática por uma reaçã̃o de nitração. $\mathrm{O}$ nitroareno resultante frequentemente um intermediário chave em sintese orgânica, podendo ser convertido num série de derivados, nomeadamente em aminas por redução. [1]

\section{Reacçōes de nitroarenos com reagentes} organomagnesianos

As reacç̃es de nitroarenos com reagentes organomagnesianos exemplificam bem a importância destes reagentes, variando mecanisticamente conforme o tipo de substrato.

\subsection{Arílicos}

A reacção dos nitroarenos com reagentes de Grignard arilicos foi primeiramente investigad por Wieland em 1903. No entanto, apenas Gilman e McCraken ${ }^{[4]}$ explicaram a formação de difenilamina, fenol e bifenil a partir da reaç̧ão de brometo de fenil-magnésio com nitrobenzeno 1 . Segundo estes autores a reacção ocorre através 1. a diç̃o 1,2 do $\mathrm{PhMgBr}$ a grupo nitro, através da deçaco 1,2 do .

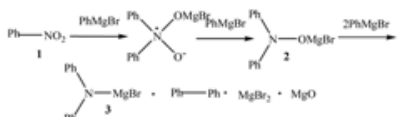

Esquema 1. Mecanismo da reaç̧ão do nitrobenzen com brometo de fenil-magnésio, proposto por Gilman e McCraken. ${ }^{|4|}$

Na realidade, a reacção de nitroarenos com halogenetos de fenil-magnésio ocorre através do mecanismo proposto por Köbrich e seus colaboradores. $\mathrm{O}$ reagente de Grignard arilico, adiciona-se a um oxigénio do grupo nitro formando 0 aducto 4, que sofre eliminação resultando na formação do composto nitroso 5 . Este sofre adição de outra molécula de reagente. Assim sendo, é a partir deste mecanismo que se forma a diaril hidroxilamino $2 \mathbf{a}^{\text {!s }}$

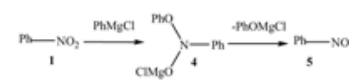

$$
\begin{aligned}
& \mathrm{Ph}_{5}-\mathrm{NO} \stackrel{\mathrm{PhM}_{2} \mathrm{CC}}{\longrightarrow} \underset{\mathrm{Ph}}{\mathrm{Ph}} \mathrm{z}_{\mathrm{2}}^{\mathrm{OM}}
\end{aligned}
$$
Esquema 2. Mecanismo da reaç̧ão do nitrobenzeno

Este tipo de reaç̧ões foi extensamente investigado, com o objectivo de obter uma sintese preparive de dis os Knecte Sapolvida apens en 2002, por Knochel e Sapountzis, uma vez que a reacçà anteriormente apresentada oferece alguma

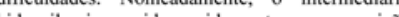
hidroxila ao ar. De facto, a diaril hidroxilamino 2, 2 a, pode ser convertida na correspondente diarilamina 6 por tratamento redutivo com $\mathrm{FeCl}_{2} / \mathrm{NaBH}_{4}$. ${ }^{|s|}$

$$
\begin{aligned}
& \text { • Raquel Gomes } \\
& \text { aluna n.'14072 de Quimica Aplicada } \\
& \text { Faculdade de Ciências e Tecnologia } \\
& \text { Monte de Caparica 2829-516 Caparica } \\
& \text { (Portugal) } \\
& \text { raquelfgomes@gmail.com }
\end{aligned}
$$

A Disciplina de Documentação e Informação é uma disciplina obrigatória, pertencente ao curriculum da licenciatura em Química Aplicada, ramo Química Orgânica da Faculdade de Ciências e Tecnologia da Universidade Nova de Lisboa. No plano curricular da licenciatura, a disciplina está incluída no $4 .^{\circ}$ ano, $1 .{ }^{\circ}$ semestre; contudo, devido ao sistema de créditos em vigor na Faculdade, é possibilitada a sua frequência aos alunos de qualquer um dos anos da licenciatura. É também uma disciplina opcional para os alunos de todas as outras licenciaturas leccionadas na Faculdade. Alunos da licenciatura em Biologia Celular e Molecular e do ramo de Biotecnologia da licenciatura em Química Aplicada frequentaram a disciplina, com muito sucesso.

A disciplina foi introduzida no campus FCT pelo Departamento de Química (DQ) no ano lectivo de 1984/1985, a convite do Prof. Doutor António Nunes dos Santos, então Professor da secção de Eng. ${ }^{a}$ Química, pelas responsáveis dos Centros de Documentação de quatro instituições, em colaboração - Eng. ${ }^{a}$ Isabel Reis do LNEC, Eng. ${ }^{a}$ Maria Odete Henriques da Quimigal, Eng. ${ }^{a}$ Manuela Azevedo da Petrogal e Eng. ${ }^{a}$ Gabriela Lopes da JNICT. No ano seguinte, a disciplina foi sub-dividida em duas: "Informação Científica e Técnica" na licenciatura em Eng. a Química e "Documentação e In-

Figura 1 "Reacções de Nitroarenos e seus Derivados com Reagentes Organomagnesianos", por Raquel Gomes n. ${ }^{\circ} 14072$

* Faculdade de Ciências e Tecnologia da Universidade Nova de Lisboa, Quinta da Torre, 2825-114 Monte da Caparica (emg@dq.fct.unl.pt) 


\section{$\underline{\text { OVERVIEW }}$}

Overview ..

1. Introduction ..

2. Interactions in catenanes.

Wals interactions............ 2

2.2 Hydrophobic interactions ............... 2

2.3 Hydrogen bonding ......................... 2

2.4 Donor-acceptor interactions ........... 3

2.5 Transition metal coordination......... 4

3. Synthetic approaches towards catenanes 4

3.1 Statistical threading approach........ 4

3.2 The Möbius strip approach.............. 4

3.3 Directed synthesis of catenanes..... 5

3.4 Templated synthesis of catenanes.. 6

References

\section{INTRODUCTION}

Organic synthesis has always tried to devise means of achieving the building of molecular systems as the result of the sequential formation of covalent bonds. ${ }^{[1]}$ However, this approach is rapidly drawing to an end, as the search for more advanced molecular topologies has firmly established itself amongst the chemical community. Furthermore, even the best nowadays chemists can not hope to build molecular systems analogous to those found in Nature, or develop the rapidly developing molecular devices (MDs) ${ }^{[2,3]}$, only with the current tools available for covalent bond forming.

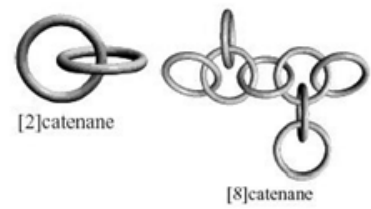

Figure $1 \rightarrow$ Examples of the structure of catenanes. Examples taken from ref.22)
Unfortunately, the synthetic chemist finds himself limited by the power of the synthetic tools available to him. ${ }^{[4]}$ This happens because nowadays a synthetic chemist has available a set of techniques and knowledge that he would not have a few years ago, which implies that nowadays' tools should allow the synthetic chemist to come up with newer and never before achieved molecular topologies. Most of these new and amazing topologies fit into group of compounds known as interlocked molecules.

Interlocked molecules consist of two or more components mechanically linked together ${ }^{[5,6,7,8]}$. One of the simplest examples of such a class of compounds are catenanes (Figure 1). Catenanes are molecules constituted by mutually interlocked rings, that can only mutually interlocked rings, that can only
be separated by the cleavage of at least be separated by the cleavage of at least
one covalent bond property, catenanes are seen as molecular aggregates and not supramolecular ones. ${ }^{[11]}$ Another property of these rings is that they have no covalent bond between them but a mechanical bond instead.

Examples of such structures have been found in nature (Figure 2) in molecules such as $\mathrm{DNA}^{[4]}$, but it was not until the 1960's that their synthesis in the laboratory was first reported. ${ }^{[10]}$
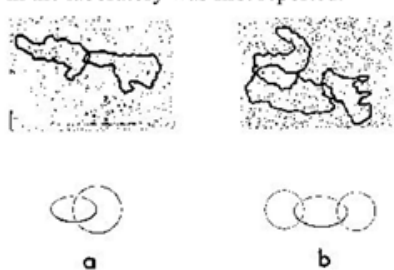

Figure $2 \rightarrow$ Electron micrographs of gyrasefrom ref. 4 .

It is the aim of this review to present a perspective of the synthetic

Documentação e Informação 2004/2005

Figura 2 "Catenanes: an overview of synthetic strategies", por Ricardo da Costa Barata, n. ${ }^{\circ} 13309$.

formação" na licenciatura em Química Aplicada.

\section{Os objectivos da disciplina são:}

1. Inventariar, descrever e dar a conhecer de forma sistematizada os tipos de fontes de informação mais relevantes - em especial no âmbito da Química Orgânica, dada a natureza dos alunos.

2. Orientar os alunos na selecção e exploração das fontes e serviços de informação.
3. Iniciar os alunos na "arte" da escrita científica.

Quando iniciam a disciplina, os alunos apenas tiveram contacto com livros de texto e, em alguns casos, com 1 ou 2 artigos científicos que utilizaram para reproduzir a parte experimental. Quando terminam a disciplina os alunos sabem distinguir as diferentes fontes e serviços de informação, a sua importância e como localizar, aprenderam a pesquisar no Beilstein, no Chemical Abstracts, conhecem bem as fontes de informação mais relevantes em Segurança Química, sabem qual a estrutura de uma patente, consultaram patentes, sabem distinguir os diferentes serviços de indexação, aprenderam a utilizá-los e sabem concretamente a diferença existente ao utilizá-los numa pesquisa científica; tiveram contacto com literatura cinzenta e qual a sua relevância, pesquisaram teses e dissertações, artigos de revisão, comunicações, full-papers, proceedings; distinguem a sua organização. Tomaram contacto com o processo de publicação científica - desde a organização dos resultados até à publicação, incluindo o processo de avaliação (referee, editor). Aprendem a avaliar a importância de uma publicação - factor de impacto das revistas, número de citações das publicações. Finalmente, aprenderam a organizar e a apresentar informação científica através da execução de um trabalho de revisão de um tema que escolheram. O impacto de tal aprendizagem é imediato, refletindo-se no modo como passam a elaborar e expor temas, relatórios e trabalhos das outras disciplinas.

Nos últimos 2 anos a disciplina sofreu um incremento qualitativo muito positivo devido aos recursos online concedidos pela Fundação para a Ciência e Tecnologia - Web of Knowledge, B-on, Portais de editores, ISI Proceedings, Current Contents, Journal Citation Reports, etc... com o acesso, em aula, às fontes e serviços de informação ou seja, ao conhecimento.

A disciplina tem os seus principais conteúdos na internet: www.dq.fct.unl.pt/ cadeiras/docinf. A consulta dos trabaIhos, entrada "Trabalhos TP" permite verificar a aprendizagem alcançada pelos alunos na disciplina e sua importância; as figuras 1 e 2 ilustram dois trabalhos de alunos da licenciatura em Química Aplicada. Para ilustrar a importância que a disciplina tem para os alunos, refiro uma citação de um aluno do ano lectivo 2003/2004 :

"Knowledge is of two kinds: we know ourselves, or we know where we can find", citação de André Filipe Costa, aluno n. ${ }^{\circ} 12022$ em "Líquidos iónicos \& as suas aplicações na reacção de DielsAlder". 